\title{
Feedback Respiratory Training to Enhance Chest Expansion and Pulmonary Function in Chronic Stroke: A Double-Blind, Randomized Controlled Study
}

\author{
Kyoung Kim, PhD, PT ${ }^{1)}$, Dennis W. Fell, MD, PT ${ }^{2)}$, JeOn HyeOng LeE, MS, PT ${ }^{1)}$ \\ 1) Department of Physical Therapy, College of Rehabilitation Science, Daegu University: \\ Jillyang, Gyeongsan, Gyeongbuk 712-714 South Korea. \\ TEL: +82 53-850-4351,FAX: +82 53-850-4351,E-mail: jeonglee@hanmail.net \\ ${ }^{2)}$ Department of Physical Therapy, University of South Alabama, Mobile, AL, USA
}

\begin{abstract}
Purpose] The purpose of this study was to investigate the effects of feedback respiratory training on chest expansion and pulmonary function in patients with stroke. [Subjects and Methods] Twenty- seven patients with stroke were randomly assigned to two groups: the feedback respiratory training group (experimental) (n=13) and the control group (control) $(\mathrm{n}=14)$. Feedback respiratory training and conventional physical therapy were performed by the experimental group. Conventional physical therapy was performed by the control group. Both groups received training three times per week for four weeks. [Results] Significant improvements were observed in chest expansion after training in the experimental group. Gains in chest expansion were greater in the experimental than in control group. Values of forced vital capacity, forced expiratory volume at one second, peak expiratory flow, vital capacity, expiratory reserve volume, and inspiratory reserve volume in pulmonary function tests increased post-training in the experimental group. With the exception of expiratory reserve volume, all of these pulmonary function test measures showed greater gains in the experimental group than in the control group. [Conclusion] Feedback respiratory training is effective for the improvement of chest expansion and pulmonary function in patients with stroke.
\end{abstract}

Key words: Feedback respiratory training, Pulmonary function, Stroke

(This article was submitted Jun. 29, 2010, and was accepted Jul. 22, 2010)

\section{INTRODUCTION}

Stroke is a leading cause of major disability in social, psychological, and physical function, and $25-30 \%$ of patients with stroke have severe motor impairment ${ }^{1)}$. Decreased motor performance occurring after a stroke limits patient activity and prolongs bed rest, and is known to decrease cardiopulmonary function in association with oxygen transport capacity ${ }^{2)}$. For patients with stroke, cardiopulmonary function is one of the important issues in preservation of life ${ }^{3)}$.

Impaired hemispheric lesions result in contralateral dysfunction of ventilatory muscles ${ }^{4)}$ and hemidiaphragmatic elevation on the normal and the affected side during deep inspiration was shown on the unaffected side ${ }^{5)}$. A small reduction in movement of the upper chest on the hemiplegic side during quiet breathing, but not of the lower chest, has also been reported. This reduction was more marked in the whole hemithorax during voluntary deep breathing ${ }^{6}$. The function of the respiratory muscles and chest wall mechanics on the paralyzed side are affected by cerebrovascular disease in hemiplegic patients ${ }^{7,8)}$. In particular, previous studies investigating pulmonary function in stroke patients have documented restrictive respiratory patterns ${ }^{9-11)}$.

Aerobic exercise for hemiplegic patients is now regarded as a necessary component of intervention to avoid cardiorespiratory problems and deconditioning after stroke $^{12,13)}$. The importance of pulmonary assessment and rehabilitation has been emphasized in the overall management of hemiplegic patients ${ }^{10)}$. Emphasis has been placed on flexibility, mobility, compliance, strength and endurance of the thoracic cage, and the trunk's primary and accessory muscles, to ensure optimal aeration of the lung fields in stroke patients ${ }^{14}$.

The feedback respiratory training device, SpiroTiger (Idiag AG, Volketswil, Switzerland), is thought to provide maximum efficacy in the improvement of respiratory function, endurance for exercise capacity, perception of dyspnea, and quality of life ${ }^{15-17)}$. Since patients with stroke may exhibit respiratory patterns, like those of restrictive lung disease patterns that are similar to those of patients with spinal cord injury, such as tetraplegia ${ }^{18-21)}$, a feedback respiratory training device for stroke patients would be 
helpful for improve chest expansion and pulmonary function. The purpose of this study was to investigate the effect of feedback respiratory training on the extent of chest expansion and pulmonary function in patients with stroke. The study tested the hypothesis that feedback respiratory training would improve chest expansion and pulmonary function in patients following a stroke.

\section{SUBJECTS AND METHODS}

\section{Subjects}

This study was approved by the institutional review board of Daegu University Hospital. Thirty participants were recruited. All subjects gave their written informed consent to participation in the experiment in accordance with the ethical standards of the Declaration of Helsinki. Clinical and demographic features of the sample are summarized in Table 1 which also shows that there were no significant differences between the groups before training.

The 30 subjects had a diagnosis of stroke based on analysis of their medical histories and brain MRI; only patients whose stroke occurred greater than six months ago were included in this study. Subjects were randomly assigned to the feedback respiratory training group (FRTG) $(n=15)$ and the control group $(C G)(n=15)$. To ensure homogeneity of variance and normal distribution for statistical reasons, three patients were excluded due to their extremely poor performance in the exercise programs; therefore, the final sample consisted of 13 FRTG (male/ female, 7/6; mean age, $57.07 \pm 6.55$ years) and 14 CG (male/female, 3/11; mean age, $57.78 \pm 5.65$ years). Patients with pulmonary disorders, severe aphasia, and impairment of cognitive function (Mini Mental Status Examination score $<24$ points) were excluded from the study.

\section{Methods}

A procedural checklist and standardized verbal instructions were used to ensure the uniformity of procedures during clinical tests. Because this was a doubleblinded experiment, patients and investigators were unaware of the test results and intervention grouping. All tests were performed before and after training. Chest expansion, in centimeters, was measured with the patient in a standing position, using a tape-measure placed circumferentially around the chest wall at the xiphoid process $^{22)}$. The chest circumference difference between maximal inspiration and expiration at the level of the xiphoid process was measured and the mean value of three measurements was calculated, similar to the method described by Viitanen ${ }^{23)}$. Subjects were asked to breathe out as much as possible while the measuring tape was drawn taut, and the chest circumference was measured at maximal expiration. The tape was then released, subjects were asked to breathe in as deeply as possible, and the circumference was measured at maximal inspiration. Values for chest expansion were obtained pre and post training.

Forced expiratory volume at one second (FEV1), forced vital capacity (FVC), ratio of forced expiratory volume at one second to forced vital capacity (FEV1)/(FVC), peak
Table 1. Baseline characteristics of study participants $(n=27)$

\begin{tabular}{lll}
\hline & FRTG & CG \\
\hline Sex (male/female) & $7 / 6$ & $3 / 11$ \\
Age (years) & $57.07 \pm 6.55$ & $57.78 \pm 5.65$ \\
Height (cm) & $164.46 \pm 8.17$ & $160.71 \pm 5.90$ \\
Weight (kg) & $61.46 \pm 8.42$ & $57.57 \pm 6.83$ \\
Paretic side (right/ left) & $6 / 7$ & $6 / 8$ \\
Etiology (Hemorrhage/Infarct) & $3 / 10$ & $2 / 12$ \\
Time since Onset (months) & $13.76 \pm 4.02$ & $13.50 \pm 2.76$ \\
Chest expansion (cm) & $4.76 \pm 1.42$ & $3.92 \pm 99$ \\
FVC (L) & $1.84 \pm .33$ & $1.77 \pm .69$ \\
FEV1 (L) & $1.69 \pm .25$ & $1.67 \pm .62$ \\
FEV1/FVC (\%) & $92.85 \pm 6.55$ & $95.16 \pm 7.54$ \\
PEF (L/S) & $3.08 \pm .95$ & $3.17 \pm 1.11$ \\
VC (L) & $2.82 \pm .34$ & $3.19 \pm .82$ \\
TV (L) & $0.51 \pm .19$ & $0.75 \pm .94$ \\
ERV (L) & $0.75 \pm .06$ & $0.71 \pm .16$ \\
IRV (L) & $1.56 \pm .27$ & $1.71 \pm .38$ \\
\hline
\end{tabular}

FRTG: Feedback Resistance Training Group; CG: Control group.

expiratory flow (PEF), vital capacity (VC), tidal volume $(\mathrm{Vt})$, inspiratory reserve volume (IRV), and expiratory reserve volume (ERV) were measured using a spirometer (Vmax 229, SensorMedics, USA). All pulmonary function measurements were taken with subjects in the sitting position and the mean value of 3 measurements was calculated.

The experimental group underwent feedback respiratory training (FRT) and conventional physical therapy (CPT) for a total of 60 minutes (FRT: $30 \mathrm{~min}, \mathrm{CPT}: 30 \mathrm{~min}$ ) a day with a 10 -minute rest break halfway through the session. This group received the training 3 days a week for 4 weeks. The control group underwent only CPT for $30 \mathrm{~min}$ a day and 3 days a week for 4 weeks.

The protocol for FRT was planned using the feedback respiratory training device known as the SpiroTiger. The SpiroTiger is used for training breathing muscles and improving pulmonary function and for providing feedback about respiratory training to patients with stroke. The SpiroTiger training device consists of a hand-held unit with a respiratory pouch and a base station. This device has a two-way piston valve connecting to a rebreathing bag. The size of the rebreathing bag was adjusted to $1.5 \mathrm{~L}$ for the level of intensity in the respiratory trainig, approximating 50 to $60 \%$ of the subject's VC as used by Gawril et al. ${ }^{15)}$, and the breathing frequency was chosen as the low frequency of 12$13 \mathrm{breath} / \mathrm{min}$ to prevent fatigue or dizziness in patients.

As the patient breathes out through the mouthpiece, the rebreathing bag stores part of the expired air. Once the rebreathing bag is filled to its capacity, a valve opens and allows the rest of the expired air to be released into the environment. The valve shuts when expiration finishes and inspiration starts. Inspiration empties the rebreathing bag first, then the valve opens and fresh outside air is inspired at the end of each inspiration. The base station monitors breathing frequency, displays visual and acoustic feedback, and stores time and data. The breathing frequency is paced by a moving light and brief sound. 
While in a sitting position, subjects held the mouthpiece to their mouth while watching the monitor of the SpiroTiger. Subjects wore a nose clip to ensure that breathing occurred exclusively through the training device. The base station was manipulated by the investigator, who pushed the start button. While watching the monitor, subjects started inspiration when the red bar reached the signal marked "in" and started expiration when the bar reached the signal marked "out". The device's display and auditory feedback are very important for constraining subjects breathing within the threshold values of isocapnia. Patients who complained about fatigue or dizziness during respiratory training were allowed to stop and rest. Respiratory training was then be restarted.

The CPT program involved a patient-specific neurofacilitation technique, planned for facilitation of symmetrical static and dynamic standing balance during walking in individuals with chronic hemiparetic stroke as a part of a regular neurorehabilitation regimen. For static balance training, patients were encouraged to shift their weight onto the paretic limb through verbal and tactile cues. A muscle strengthening exercise for the gluteus medius muscles was also performed to improve eccentric controlled mobility of the pelvis during the stance phase of gait and to prevent pelvic drop. For dynamic balance training, kinesthetic tactile inputs in relation to pelvic rotation and weight shifting were provided during gait training ${ }^{24)}$.

Independent variables were the forms of intervention $($ FRT + CPT, CPT $)$. Dependent variables, including the measure of chest expansion between maximal inspiration and expiration and values from pulmonary function testing, were recorded before and after training. Statistical comparisons of chest expansion and pulmonary function before and after training within each group were made with the paired samples t-test. Comparisons of post training gains of chest expansion and pulmonary function between FRTG and CG were accomplished with the independent samples ttest. The statistical software, SPSS 17.0 (SPSS, Chicago, IL, USA), was used for statistical analyses.

\section{RESULTS}

No significant differences in baseline characteristics were observed between the two groups. Changes in chest expansion (Table 2) and pulmonary function (Table 3 ) are summarized for the two groups before and after training. The FRTG showed a significant increase in chest expansion after training $(\mathrm{p}<0.05)$. There was also a significant difference in post-training gains between the FRTG and the $\mathrm{CG}(\mathrm{p}<0.05)$. However, the CG demonstrated no significant changes in chest expansion after training $(\mathrm{p}>0.05)$.

Statistically significant post-training improvements in pulmonary function, including FVC, FEV1, PEF, VC, ERV, and IRV were detected in the FRTG $(\mathrm{p}<0.05)$. Comparison of the gains between the two groups showed significant differences in FVC, FEV1, PEF, VC, and IRV $(\mathrm{p}<0.05)$. However, there were no significant differences in the posttraining values of FEV1/FVC, TV, and ERV between the two groups $(\mathrm{p}>0.05)$.

\section{DISCUSSION}

The specific aim of this randomized controlled study was to demonstrate that FRT in addition to a CPT program has a more beneficial effect on chest expansion and pulmonary function performance than a CPT program in patients with stroke. The study results indicate larger and more efficient chest expansion and pulmonary function with FRT than without. This result supports the primary hypothesis of the study that FRT would improve chest expansion and pulmonary function in people following a stroke.

The chest expansion data showed significant improvement in the FRT group when compared with gains in the control group, indicating an augmented therapeutic effect of FRT. In this study, FRT included repetitive, continuous performance of maximal inspiration and expiration, and inspiratory muscle training may have enhanced chest expansion in the stroke patients. The normal value of chest expansion in healthy people between the ages of 55 and 64 is between 4.0 and $5.5 \mathrm{~cm}^{22)}$. However, in this study, the chest expansion value of pre-training stroke patients was slightly lower, between 3.9 and $4.7 \mathrm{~cm}$. Values of chest expansion increased by $22.7 \%$ after FRT within FRTG, whereas control group participants showed little change in their values of chest expansion. These results are similar to findings recorded after inspiratory muscle training in patients with COPD and myasthenia gravis ${ }^{25,26)}$, and they demonstrate increased chest expansion after inspiratory muscle training. Interestingly, Sahin et al. ${ }^{27)}$ reported a positive correlation between chest expansion and maximal inspiratory pressure (MIP) in patients with ankylosing spondylolitis. Moreover, displacement of the ribcage in the lateral outward direction during inspiration in normal subjects using an automatic motion analyzer was greater than the displacement during tidal breathing ${ }^{28)}$, and lateral movement of the ribcage at the level of the 4th rib during inspiration was increased in the neutral posture using an electromagnetic tracking device in healthy subjects ${ }^{29)}$. Thus, these findings support the use of FRT as an effective chest expansion method for patients following a stroke.

The pulmonary function data in this study showed significant improvements in FVC, FEV1, PEF, VC, and IRV of the FRT group while there were no significant improvements in the control group. This finding could be attributed to the FRT which trained subjects in maximal inspiration and expiration with a regular breathing frequency dictated by a SpiroTiger.

FVC values within the FRT group increased by $19.6 \%$, whereas participants in the CG showed no significant change in FVC values between pre- and post-training. This finding seems to strongly support a positive effect of FRT. The greater FVC value is likely the result of improved inspiration after FRT. The present findings are similar to the findings of a previous study ${ }^{16}$ ) that demonstrated that the FVC value of cystic fibrosis patients increased by $5.7 \%$ when they used a SpiroTiger. Scherer et al. ${ }^{30)}$ reported increased FVC (\% predicted value) during respiratory muscle training with a SpiroTiger, as compared to the use of an incentive spirometer in patients with COPD. In a sample 
Table 2. Comparison of change in chest expansion between the training and control groups

\begin{tabular}{llllll}
\hline & FRTG & & & CG & \\
\cline { 2 - 3 } \cline { 5 - 6 } & Pretest & Posttest & & Pretest & Posttest \\
\hline $\begin{array}{lllll}\text { Chest expansion presented } \\
\text { as mean } \pm \text { standard deviation }(\mathrm{cm})\end{array}$ & $4.76 \pm 1.42$ & $5.84 \pm 1.57^{* \mathrm{a}}$ & & $3.92 \pm .99$ & $3.71 \pm 1.13^{\mathrm{a}}$ \\
\hline
\end{tabular}

FRTG: Feedback respiratory training group; CB: Control Group. *significant difference from pre-test at $\mathrm{p}<0.05$.

a significant. difference in gains between the two groups at $\mathrm{p}<0.05$.

Table 3. Comparison of change in pulmonary function in the training and control groups with values presented as mean \pm standard deviation

\begin{tabular}{llllll}
\hline & FRTG & & & \multicolumn{2}{l}{ CG } \\
\cline { 2 - 3 } \cline { 5 - 6 } & Pretest & Posttest & & Pretest & Posttest \\
\hline FVC (L) & $1.84 \pm .33$ & $2.20 \pm 25^{* a}$ & & $1.77 \pm .69$ & $1.75 \pm .73^{\mathrm{a}}$ \\
FEV1 (L) & $1.69 \pm .25$ & $2.03 \pm .28^{* \mathrm{a}}$ & & $1.67 \pm .62$ & $1.67 \pm .65^{\mathrm{a}}$ \\
FEV1/FVC (\%) & $92.8 \pm 6.6$ & $92.1 \pm 5.6$ & & $95.2 \pm 7.5$ & $96.2 \pm 5.4$ \\
PEF (LPS) & $3.08 \pm .95$ & $3.75 \pm 73^{* a}$ & & $3.17 \pm 1.11$ & $3.20 \pm 1.13 \mathrm{a}$ \\
VC (L) & $2.82 \pm .34$ & $3.28 \pm .51^{* \mathrm{a}}$ & & $3.19 \pm .82$ & $3.01 \pm .57^{\mathrm{a}}$ \\
TV (L) & $0.51 \pm .19$ & $0.71 \pm .41$ & & $0.75 \pm .94$ & $0.50 \pm .21$ \\
ERV (L) & $0.75 \pm .06$ & $0.85 \pm .09^{*}$ & & $0.71 \pm .16$ & $0.77 \pm .13$ \\
IRV (L) & $1.56 \pm .27$ & $1.71 \pm .23^{* a}$ & & $1.71 \pm .38$ & $1.75 \pm .36^{\mathrm{a}}$ \\
\hline
\end{tabular}

*significant difference from pre-test at $<0.05 .{ }^{a}$ significant difference in gains between two groups at $\mathrm{p}<0.05$. FRTG: Feedback respiratory training group; CB: Control Group. FVC: forced vital capacity; FEV1: forced expiratory volume at one second. PEF: peak expiratory flow; VC: vital capacity; TV: tidal volume. ERV: expiratory reserve volume; IRV: inspiratory reserve volume.

with stroke, Sutbeyaz et al. ${ }^{\text {s) }}$ reported increased FVC after inspiratory muscle training. According to the American Thoracic Society, characteristics of restrictive lung disease patterns include a normal or an increase in the normal FEV1/FVC ratio, and a decrease of the normal FVC value $^{32}$. Reference values for spirometry include a FEV1/ FVC ratio of at least $79 \%$ and a FVC of $4.5 \mathrm{~L}^{33}$. The stroke patients in this study had pre-training values indicating a normal FEV1/FVC of over $79 \%$ and a decreased $\mathrm{FVC}$ of $1.84 \mathrm{~L}$. This finding supports a respiratory pattern of restrictive lung disease in the individuals with stroke.

In this study, the FEV1 value was 1.69 liters before respiratory training. This finding is similar to that of the respiratory pattern of restrictive lung disease because Miller ${ }^{32)}$ reported that FEV1 value in normal persons is 4.0 liter and the value in restrictive lung disease subjects is approximately 1.5 liter. In the FRT group, the FEV1 value increased by $20 \%$ after respiratory training. This change may be the result of better performance of expiration, through increased tension on the chest wall, induced by training the diaphragm and other inspiratory muscles with a FRT device ${ }^{16,30)}$. In previous studies, Liaw et al. ${ }^{19)}$ documented a significant change in FEV1 following resistive inspiratory muscle training in patients with acute cervical cord injury. Patients showed an increase of $31 \%$ in FEV1 that occurred within 6 weeks of training. Zupan et al. ${ }^{34)}$ also reported that FEV1 values increased by $21 \%$ following maximal sustained inspiration for 20-30 min, twice a day, 6 days a week, for 4 weeks in patients with tetraplegia. Moreover, an inspiratory muscle training program for stroke patients resulted in improved FEV1, which appeared to help decrease stroke recurrence, because low respiratory muscle strength is associated with an increased risk of stroke ${ }^{31)}$. The FRT performed in this study appears to be appropriate for improving pulmonary function in patients with stroke.

Compared to the post-training PEF value of the control group, which did not significantly improve, the average post-training PEF values of the FRT group increased by $21 \%$, perhaps as a result of continuous expiration performance with the FRT device. This finding is similar to the findings of a previous study ${ }^{31)}$ that demonstrated that since PEF is effort-dependent, improved PEF values may reflect an increase in muscle strength and support the presence of effective cough in stroke patients. Scherer et al. ${ }^{30)}$ reported that maximal expiratory pressure after respiratory muscle training with the SpiroTiger device was increased in patients with COPD, and that expiratory muscle strengthening may result in an effective cough and prevent pneumonia and microatelectasis. In the current study, the improved PEF value seems to provide strong support for a positive effect of FRT and may decrease the risk of respiratory infection. VC values in the FRT group increased by $16.3 \%$ after training, whereas control group participants showed little change in VC value between pre- and posttraining. This finding is similar to findings from a previous study $^{31)}$, which demonstrated increased VC in a sitting position, after using a threshold inspiratory muscle trainer, in patients with stroke, providing strong support for a positive effect of FRT, including inspiration. Mansel et al. ${ }^{35)}$ 
documented that evaluation of lung function in patients with SCI showed a respiratory pattern of restrictive lung disease, and patients performing resistive inspiratory muscle training showed improvements in $\mathrm{VC}$ of up to $67 \%{ }^{19)}$.

The IRV in the FRT group increased by $9.6 \%$ after training. This improvement may be the positive result of a training effect of SpiroTiger on inspiratory muscles. This finding is similar to findings from previous studies ${ }^{20,36)}$, which demonstrated increased IC and MIP values. Enright et al. ${ }^{36)}$ reported that high intensity inspiratory muscle training resulted in significantly increased VC, total lung capacity, and MIP in healthy subjects. The IRV value is closely associated with the $\mathrm{VC}$ value because $\mathrm{VC}$ is the sum of IRV, TV, and ERV. An increase in vital capacity through respiratory training, as with SpiroTiger, may increase IRV. The normal reference value for IRV has been reported as 3 liters $^{32}$, but in this study the value was lower (Table 3 ), consistent with the restrictive respiratory pattern expected in stroke patients. In addition, Loveridge et al. ${ }^{20)}$ performed resistive inspiratory muscle training in patients with quadraplegia and reported that $\mathrm{VC}$ and MIP were increased by $44 \%$ in the experimental group.

The current study had several limitations, including a lack of any measurement of respiratory muscle strength, a relatively small sample size of patients with stroke, and a short duration of training. Further investigation with a larger sample size and long-term training is needed. In a future study, a cross-over design in which the control group would switch in phase II to receive the experimental intervention would also be useful. Furthermore, another potential limitation is the simplicity of the chest expansion measurement with a tape-measure used in this study. Other machines, including those used for three dimensional motion analysis, could perhaps provide better measurement of ribcage movements. Further research on the relationship between various breathing exercises and ribcage movements in people with stroke will be necessary, particularly an investigation of the differences between the left and right side of the ribcage with various interventions.

\section{ACKNOWLEGEMENT}

This research was supported by a Daegu University Research Grant, 2007.

\section{REFERENCES}

1) Duncan PW, Horner RD, Reker DM, et al.: Adherence to postacute rehabilitation guidelines is associated with functional recovery in stroke. Stroke, 2002, 33: 167-177.

2) Ferretti G, Girardis M, Moia C, et al.: Effects of prolonged bed rest on cardiovascular oxygen transport during submaximal exercise in humans. Eur J Appl Physiol Occup Physiol, 1998, 78: 398-402.

3) Carr M, Jones J: Physiological effects of exercise on stroke survivors. Top Stroke Rehabil, 2003, 9: 57-64.

4) De Troyer A, Zegers De Beyl D, Thirion M, et al.: Function of the respiratory muscles in acute hemiplegia. Am Rev Respir Dis, 1981, 123: 631-632.

5) Cohen E, Mier A, Heywood P, et al.: Diaphragmatic movement in hemiplegic patients measured by ultrasonography. Thorax, 1994, 49: 890-895.

6) Fluck DC: Chest movements in hemiplegia. Clin Sci, 1966, 31: 383-388.

7) Annoni JM, Ackermann D, Kesselring J, et al.: Respiratory function in chronic hemiplegia. Int Disabil Stud, 1990, 12: 70-80.

8) Lanini B, Bianchi R, Romagnoli I, et al.: Chest wall kinematics in patients with hemiplegia. Am J Respir Crit Care Med, 2003, 168: 109-113.

9) Fugl-Meyer AR, Linderholm H, Wilson AF, et al.: Restrictive ventilatory dysfunction in stroke: its relation to locomotor function. Scandinavian journal of rehabilitation medicine Supplement, 1983, 9: 118-124.

10) Haas A, Rusk HA, Pelosof $\mathrm{H}$, et al.: Respiratory function in hemiplegic patients. Arch Phys Med Rehabil, 1967, 48: 174-179.

11) Odia GI: Spirometry in convalescent hemiplegic patients. Arch Phys Med Rehabil, 1978, 59: 319-321.

12) Lee $\mathrm{CD}$, Blair $\mathrm{SN}$ : Cardiorespiratory fitness and stroke mortality in men. Med Sci Sports Exerc, 2002, 34: 592-595.

13) Sezer N, Ordu NK, Sutbeyaz ST, et al.: Cardiopulmonary and metabolic responses to maximum exercise and aerobic capacity in hemiplegic patients. Funct Neurol, 2004, 19: 233-238.

14) Narain S, Puckree T: Pulmonary function in hemiplegia. Int J Rehabil Res, 2002, 25: 57-59.

15) Markov G, Spengler CM, Knöpfli-Lenzin C, et al.: Respiratory muscle training increases cycling endurance without affecting cardiovascular responses to exercise. Eur J Appl Physiol, 2001, 85: 233-239.

16) Sartori R, Barbi E, Poli F, et al.: Respiratory training with a specific device in cystic fibrosis: a prospective study. J Cyst Fibros, 2008, 7: 313-319.

17) Spengler CM, Roos M, Laube SM, et al.: Decreased exercise blood lactate concentrations after respiratory endurance training in humans. Eur J Appl Physiol Occup Physiol, 1999, 79: 299-305.

18) Fugl-Meyer AR, Grimby G: Respiration in tetraplegia and in hemiplegia: a review. Int Rehabil Med, 1984, 6: 186-190.

19) Liaw MY, Lin MC, Cheng PT, et al.: Resistive inspiratory muscle training: its effectiveness in patients with acute complete cervical cord injury. Arch Phys Med Rehabil, 2000, 81: 752-756.

20) Loveridge B, Badour M, Dubo H, et al.: Ventilatory muscle endurance training in quadriplegia: effects on breathing pattern. Paraplegia, 1989, 27: 329-339.

21) Mueller G, Perret C, Spengler CM: Optimal intensity for respiratory muscle endurance training in patients with spinal cord injury. J Rehabil Med, 2006, 38: $381-386$

22) Moll JM, Wright V: An objective clinical study of chest expansion. Ann Rheum Dis, 1972, 31: 1-8.

23) Viitanen JV, Kautiainen H, Suni J, et al.: The relative value of spinal and thoracic mobility measurement in ankylosing spondylitis. Scand J Rheumatol, 1995, 24: 94-97.

24) O'Sullivan SB: Stroke. In: O'Sullivan SB, Schmitz TJ (eds): Physical Rehabilitation, (5th ed): Philadelphia: F.A. Davis Company, 2007, pp705-775.

25) Fregonezi GA, Resqueti VR, Güell R, et al.: Effects of 8-week, interval-based inspiratory muscle training and breathing retraining in patients with generalized myasthenia gravis. Chest, 2005, 128: 1524-1530.

26) Minoguchi H, Shibuya M, Miyagawa $T$. et al.: Cross-over comparison between respiratory muscle stretch gymnastics and inspiratory muscle training. Intern Med, 2002, 41: 805-812.

27) Sahin G, Calikoglu M, Ozge C, et al.: Respiratory muscle strength but not BASFI score relates to diminished chest expansion in ankylosing spondylitis. Clin Rheumatol, 2004, 23: 199-202.

28) De Groote A, Wantier M, Cheron G, et al.: Chest wall motion during tidal breathing. J Appl Physiol, 1997, 83: 1531-1537.

29) Lee LJ, Chang AT, Coppieters MW, et al.: Changes in sitting posture induce multiplanar changes in chest wall shape and motion with breathing. Respir Physiol Neurobiol, 2010, 170: 236-245.

30) Scherer TA, Spengler CM, Owassapian D, et al.: Respiratory muscle endurance training in chronic obstructive pulmonary disease: impact on exercise capacity, dyspnea, and quality of life. Am J Respir Crit Care Med, 2000, 162: 1709-1714.

31) Sutbeyaz ST, Koseoglu F, Inan L, et al.: Respiratory muscle training improves cardiopulmonary function and exercise tolerance in subjects with subacute stroke: a randomized controlled trial. Clin Rehabil, 2010, 24: 240-250.

32) Miller A: Lung function testing: selection of reference values and interpretative strategies. Am Rev Respir Dis, 1992, 146: 1368-1369.

33) Youtsey J: Basic pulmonary function measurements. In: Egan's Fundamentals of Respiratory Therapy. St Louis: Mosby, 1982, pp 170-190.

34) Zupan A, Savrin R, Erjavec T, et al.: Effects of respiratory muscle training and electrical stimulation of abdominal muscles on respiratory capabilities in tetraplegic patients. Spinal Cord, 1997, 35: 540-545.

35) Mansel JK, Norman JR: Respiratory complications and management of spinal cord injuries. Chest, 1990, 97: 1446-1452.

36) Enright SJ, Unnithan VB, Heward C, et al.: Effect of high-intensity inspiratory muscle training on lung volumes, diaphragm thickness, and exercise capacity in subjects who are healthy. Phys Ther, 2006, 86: 345-354. 